\title{
Diagnóstico y tratamiento de las várices esófago gástricas en Chile: Realidad nacional
}

\author{
Ling $\operatorname{Vargas} \mathrm{T}^{1}$, Loreto 0 valle $\mathrm{A}^{1}$, Ximena Morales $0^{1}$, \\ Solange Agar $F^{1}$, René Estay $0^{1}$, Juan Ramón Soto $H^{1}$, \\ Rodolfo Armas $\mathbf{M}^{1}$, María Teresa Vergara $A^{2}$, Roberto Nazal $S^{3}$. \\ Management of bleeding \\ esophageal varices in public \\ and private institutions in Chile
}

Background: The better treatment modalities for bleeding esophageal varices have improved the prognosis of cirrhosis. Aim: To inquire about diagnostic and treatment modalities for esophageal bleeding in Chile. Material and methods: An enquiry about diagnosis and treatment of esophageal bleeding was designed and electronically sent to public and private health institutions that could admit patients and were located in cities with more than 100,000 inhabitants. Results: The enquiry was answered by 31 of 35 public and 17 of 19 private health institutionis that were consulted. Emergency endoscopy was available in 6 of 27 public and in the 16 private institutionis that had an emergency room. Rubber band ligation was available in 16 public (52\%) and in all private institutions. Cyanoacrylate injections were done in 10 public (32\%) and $11(65 \%)$ private institutions. No public institution installed transjugular intrahepatic portosystemic shunts, but 8 had occasional access to this technique. This procedure was done in 7 (41\%) private institutions and all had access to it. Surgical treatment was feasible in 20 public (65\%) and all private institutions. Primary prophylaxis was done in 18 public (58\%) and 14 private (82\%) institutions. Secondary prophylaxis was carried out in 26 public (84\%) and 16 private (94\%) institutions. Conclusions: Public health institutions have poor access to adequate diagnostic and treatment methods for esophageal bleeding. The primary and secondary prophylaxis of esophageal varices must be improved in both types of institutions (Rev Méd Chile 2008; 136: 837-43).

(Key words: Cyanoacrylates; Esophageal and gastric varices; Gastrointestinal hemorrhage)

Recibido el 27 de junio, 2007. Aceptado el 1 de abril, 2008.

${ }^{1}$ Hospital San Juan de Dios, Universidad de Chile, ${ }^{2}$ Hospital Naval de Viña del Mar. ${ }^{3}$ Hospital San José, Santiago de Chile.

Conflictos de intereses. Los autores no tienen auspicio de los fabricantes de endoscopios.

Correspondencia a: Dr. Ling Vargas Tank. Alvar Aalto 1271.

Vitacura. Santiago. Fax: (2)4928245.

E mail: lvatank@hotmail.com 
L a a hemorragia por ruptura de várices esófago gástricas (HVEG) es una importante causa de descompensación y muerte en individuos portadores de cirrosis hepática. En ausencia de tratamiento, la primera HVEG encierra un riesgo de morir que fluctúa entre $30 \%$ y $50 \%$, dejando a los sobrevivientes proclives a nuevos sangrados ${ }^{1}$. El desarrollo tecnológico alcanzado en los últimos decenios permite detener y prevenir el sangrado por várices esofágicas (VE) y gástricas (VG). Hacer profilaxis de la hemorragia ha incrementado la expectativa de vida de estos pacientes ${ }^{2,3}$. En Chile las nuevas tecnologías se han introducido desigualmente en los diversos centros asistenciales públicos o privados, donde los pacientes consultan dependiendo de su sistema de previsión, sin conocer el grado de desarrollo tecnológico de ellas y sin posibilidades de cambiar su sitio de atención.

El objetivo de este trabajo fue conocer cómo se diagnostica y trata a los pacientes que presentan una HVEG en las instituciones públicas (IPU) y privadas (IPR) en Chile. En particular, la posibilidad de realizar una endoscopia expedita en las instituciones con Servicio de Urgencia adosado, y la de aplicar las nuevas tecnologías endoscópicas de hemostasis para VEG. Se investigó además la disposición a realizar profilaxis primaria y secundaria en los diversos establecimientos.

\section{MÉTODO}

Se diseñó una encuesta que permitiera conocer cómo se diagnosticaba y trataba los pacientes con HVEG (Tabla 1). No se pretendió evaluar la infraestructura general de los hospitales.

La encuesta se concretó electrónicamente entre septiembre de 2004 y marzo de 2005, considerando las instituciones de salud que disponían de hospitalización y se ubicaban en ciudades de más de 100.000 habitantes. Fueron incluidas Arica, Iquique, Antofagasta, Calama, Copiapó, La Serena, Ovalle, Coquimbo, Valparaíso, Viña del Mar, Quilpué, Santiago, Rancagua, Curicó, Talca, Chillán, Los Ángeles, Concepción, Talcahuano, Temuco, Valdivia, Osorno, Puerto Montt, Coyhaique y Punta Arenas ${ }^{4}$. Coyhaique, no obstante ser una ciudad menos poblada (población 50.000 habitantes aproximadamente), se incluyó por su aisla- miento geográfico. Se seleccionaron para ser encuestados 35 centros asistenciales de la red sanitaria del Estado y 19 privados propiamente tales, o que se comportaban como tales en lo referente a acceso y cobros, como es el caso de los Hospitales Universitarios y los de las FFAA y de Orden.

El cuestionario se dirigió a los médicos jefes de unidades de gastroenterología o endoscopia. En algunos casos se repitió la encuesta a otros médicos del grupo para ratificar la información.

Los resultados se analizaron aplicando el test exacto de Fisher.

\section{Resultados}

Se consiguió respuesta de 31 de las 35 IPU encuestadas: los hospitales de Iquique, Antofagasta, Calama, Copiapó, La Serena, Coquimbo, Viña del Mar, Quilpué, Rancagua, Curicó, Talca, Chillán, Los Ángeles, Concepción, Talcahuano, Temuco, Valdivia, Osorno, Coyhaique y Punta Arenas. En Valparaíso los hospitales Van Buren y Pereira y en Santiago los hospitales Barros Luco-Trudeau, Félix Bulnes, Padre Hurtado, Posta Central, Salvador, San Borja Arriarán, San José, San Juan de Dios y Sótero del Río. No se obtuvo respuesta oportuna de los hospitales de Arica, Ovalle, Puerto Montt y Parroquial de San Bernardo.

Se recibió respuesta de 17 de las 19 IPR encuestadas: los hospitales de las Universidades Católica y de Chile, Militar, Naval de Viña del Mar y de Talcahuano, de la Fuerza Aérea y DIPRECA y de Carabineros en Santiago; Clínicas Alemana, Dávila, Indisa, Las Condes, Santa María y Tabancura de Santiago; Reñaca de Viña del Mar y Alemana de Temuco y Osorno. No se obtuvo oportunamente la información de los hospitales del Profesor de Santiago y FUSAT de Rancagua.

Atención de urgencia y recursos endoscópicos. En 27 IPU ingresaban pacientes a través de un Servicio de Urgencia las 24 horas del día; pero sólo en 5 se disponía de un sistema de endoscopia de urgencia: 4 en provincia y una en la Región Metropolitana. En un IPU se podía solicitar endoscopias a una IPR con relativa facilidad. En todas las demás la endoscopia funcionaba sólo durante algunas horas y en días hábiles. 


\section{Tabla 1. Encuesta N acional. H emorragia digestiva (H D ) por várices esófago gástricas (VEG) e hipertensión portal}

Refiérase a la institución en la que Ud. trabaja:

I. Atención de pacientes con HD.

1. Atención de urgencia y recurso endoscópico.

a. El ingreso de pacientes con HD se produce:

- Durante 24 horas 365 días año.

- Sólo en horario hábil.

b. Si se recibe individuos con HD los 365 días, la endoscopia es realizada:

- Inmediatamente después de conseguida la estabilidad hemodinámica.

- Sólo en horario hábil.

c. Si no se dispone de endoscopia de urgencia los pacientes con HD activa son derivados a otro centro:

- Siempre o habitualmente.

- En casos especiales.

- Nunca.

2. Para detener una hemorragia por várices esofágicas dispone de:

a. Ligadura elástica:

- Sí.

- En forma interrumpida. - No.

b. Esclerosis:

- Sí.

- No.

c. Balón de Sengstaken:

- Sí.

- No.

3. Para detener una hemorragia por várices gástricas dispone de:

a. Inyección de cianoacrilato:

- Sí.

- No.

4. Disponibilidad de recursos de excepción en el tratamiento de la HD por VEG.

a. Si no logra detener una HD por VEG por técnicas endoscópicas, citiene Ud. acceso a TIPS?

- Sí. En su hospital. $\quad$ - No; pero puede derivar a otro centro. - No.

b. Considera que puede recurrir a cirugía para controlar un sangrado incohersible:

- Sí.

- No.

II. Se hace profilaxis de la recidiva hemorrágica en pacientes que sangraron por VEG.

- Sí (- endoscópica. - con propanolol. - mixta). - No se hace.

III. Busca y trata várices esófago gástricas en cirróticos para hacer profilaxis primaria del sangrado.

- Se buscan y tratan siempre o casi siempre.

- Se hace sin regularidad.

- Ha utilizado ligaduras elástica en la profilaxis de la primera hemorragia:

- Sí.

- No se hace.

- No.

Experiencia en Hospital Público/Hospital Universitario/Hospital FFAA/Clínica Privada.

(Médico que responde)

En 16 IPR se disponía de Servicio de Ungencia adosado y en todas era posible realizar endoscopias las 24 horas del día (Tabla 2).

En las 4 IPU y la IPR que no contaban con Servicio de Urgencia adosado, se carecía de un sistema para hacer endoscopia de urgencia. En caso de que alguno de los enfermos sangrara, sólo en la IPR se podía derivar en horario no hábil los pacientes a una institución vecina.

Recursos disponibles para detener la hemorragia digestiva por VE y VG se muestran en la Tabla 3. 
Tabla 2. D isponibilidad de endoscopia de urgencia en instituciones públicas y privadas de salud que funcionan con Servicio de U rgencia adosado y que reciben pacientes con hemorragia digestiva los 365 días del año, septiembre 2004 a marzo 2005

\begin{tabular}{|lccc|}
\hline Instituciones de salud & Públicas & Privadas & Valor de $\mathrm{p}$ \\
\hline Totales & $\mathrm{n}=27(\%)$ & $\mathrm{n}=16(\%)$ & \\
Disponen de endoscopia de urgencia & $5(18,5)$ & $16(100)$ & $<0,0001$ \\
Consiguen endoscopia de urgencia & $1(3,7)$ & $0(0)$ & $<0,0001$ \\
Sólo disponen de endoscopia en horario hábil & $21(77,8)$ & & \\
\hline
\end{tabular}

Profilaxis de la recidiva hemorrágica en pacientes que sangraron por VEG. En 26 IPU $(83,9 \%)$ se realizaba algún grado de profilaxis: en 17 exclusivamente endoscópica, en 4 con propanolol y en 5 con una alternativa mixta. En 5 no se realizaba profilaxis secundaria. En 16 IPR (94,1\%) se hacía profilaxis: en 14 exclusivamente endoscópica, y en 2 con terapéutica mixta. En 1 no se realizaba profilaxis secundaria.

Profilaxis primaria del sangrado por VEG. En 18 (58,1\%) IPU se buscaba regularmente VEG en pacientes con cirrosis para realizar profilaxis primaria, en 8 esto se hacía en forma poco sistemática y en 5 no se investigaba.
En $14(82,4 \%)$ de las IPR se buscaba habitualmente VEG para realizar profilaxis primaria; en una de ellas en forma planificada. En las otras 3 esto se hacía en forma poco sistemática.

En 16 IPU y en 12 IPR se había practicado profilaxis primaria de la hemorragia por VEG mediante ligaduras.

\section{DisCUSIÓN}

El número de hospitalizaciones en relación a una HVEG en Chile, aunque desconocido, debería ser varias veces mayor que el número de muertes por esta causa ${ }^{5}$. Por otro lado, si consideramos que

Tabla 3. Técnicas disponibles para detener la hemorragia por várices esófago gástricas en instituciones públicas y privadas de salud, septiembre 2004 a marzo 2005

\begin{tabular}{|lccc|}
\hline Instituciones de salud & Públicas & Privadas & Valor de $\mathrm{p}$ \\
\hline Totales & $\mathrm{n}=31(\%)$ & $\mathrm{n}=17(\%)$ & \\
Esclerosis & $28(90,3)$ & $17(100)$ & $\mathrm{ns}$ \\
Ligadura elástica & & & \\
$\quad$ Disponible en forma interrumpida & $9(29)$ & & \\
$\quad$ Disponible habitualmente & $16(51,6)$ & $17(100)$ & $<0,0003$ \\
Inyección de cianoacrilato & $10(32,2)$ & $11(64,7)$ & $<0,03$ \\
Balón de Sengstaken & $31(100)$ & $17(100)$ & $\mathrm{ns}$ \\
TIPS & 0 & $7(41,1)$ & $<0,0003$ \\
$\quad$ Instalación en la misma institución & $8(25,8)$ & $17(100)$ & $<0,0001$ \\
$\quad$ Posibilidad de acceder en otra & $20(64,5)$ & $17(100)$ & $<0,004$ \\
Cirugía & &
\end{tabular}


alrededor de la mitad de los 4.000 portadores de cirrosis o tumores hepáticos que mueren en Chile anualmente, muy probablemente lo hacen por una hemorragia digestiva, podemos vislumbrar el importante número de ingresos que determina esta patología 6 .

En la actualidad, el tratamiento de los pacientes que sangran por VEG incluye endoscopia de urgencia y acceso a procedimientos como ligadura elástica, inyección de cianoacrilato o administración de glisilpresina endovenosa, TIPS o cirugía. La inyección de esclerosantes y el uso de balones son excepcionales ${ }^{2}$.

Este trabajo deja en evidencia importantes limitaciones en la implementación de la endoscopia de urgencia y en la del tratamiento de las HVEG en las IPU al compararlas con las IPR. La explicación a este fenómeno es sociopolítica y escapa al estudio. La corrección de esta situación, a su vez, es compleja e implicaría adquirir equipamiento, entrenar especialistas y estructurar y financiar equipos que cubrirían turnos. Una solución de fondo muy probablemente va a variar dependiendo del tamaño de las ciudades y del desarrollo local de la endoscopia. En comunidades pequeñas podría establecerse convenios con los médicos que allí residen; pero, en las más extensas, como en la Región Metropolitana o la V Región, por ejemplo, el camino necesariamente implicaría estructurar un sistema de turnos. La organización de turnos de llamada por institución, a su vez, no parece práctica por el número de especialistas que se necesitarían para satisfacer una demanda muy dispersa. Por otro lado, la concentración de pacientes en una gran unidad de endoscopia en una IPU, o su derivación a una o más IPR, tendría el inconveniente de incrementar el traslado de pacientes graves, hemodinámicamente inestables y potencialmente quirúrgicos por ciudades cada vez más complejas de transitar.

Es posible que una "Unidad de Endoscopia Móvil", dotada de recursos humanos y de implementación técnica suficiente, puesta a disposición de IPU y privadas, pudiera ser una solución eficiente y menos onerosa al dar servicio a numerosos centros asistenciales. Por cierto que una organización de esa naturaleza podría cubrir otras emergencias de resolución endoscópica, como son la extracción de cuerpos extraños y tratamiento de hemorragias no variceales, entre otras.

En el campo de la terapéutica endoscópica, la ligadura elástica es la técnica más utilizada en los pacientes que sangran por VE. La primera experiencia publicada en Chile con su uso data de 2001 y se originó en una IPU7. Llama la atención que en la actualidad se acceda rutinariamente a ligadura sólo en la mitad de las IPU versus el 100\% de las IPR. Esta situación no parece explicarse por una simple falta de recursos, particularmente si se tiene en cuenta que otro $30 \%$ de las IPR tiene acceso esporádico a esa técnica. La limitación es muy probablemente sólo de organización, ya que esos adminículos son fácilmente reutilizables. Cabe tener presente que no contar con ligaduras elásticas obliga a ofrecer a los pacientes la esclerosis de VE pese a su mayor incidencia de complicaciones, ${ }^{8}$ lo que obviamente puede resultar en un servicio peor y de mayor costo.

Un procedimiento menos difundido es la inyección de cianoacrilato en várices gástricas. La primera comunicación nacional del uso del cianoacrilato se origina en 1999, también en una IPU ${ }^{9}$. Su incorporación ha sido aún más lenta que la de la ligadura, lo que puede explicarse por el extendido e infundado temor a dañar el instrumento con pegamento $\mathrm{y}$, muy probablemente, por la menor incidencia de esta forma de sangrado variceal, lo que reduce las posibilidades de formarse una experiencia. La incorporación particularmente baja de esta técnica en las IPU puede deberse al costo que implica desechar inyectores y adquirir insumos relativamente caros. En los próximos años se podrá evaluar si los cursos prácticos implementados recientemente a nivel nacional han logrado estimular el empleo de esta valiosa técnica.

Los tratamientos farmacológicos que reducen la presión portal se han desarrollado en paralelo a los endoscópicos y no fueron considerados en esta encuesta. Esta alternativa terapéutica ha sido poco utilizado en el país. Una serie nacional objetivó que la esclerosis era mejor que el octeótrido en el control del sangrado ${ }^{10}$. Los resultados comunicados con glisil presina ${ }^{11}$ hacen interesante la evaluación del rol que pudiera jugar este fármaco en la reducción del requerimiento de endoscopia de urgencia ${ }^{12}$.

La instalación de TIPS es el tratamiento de elección de segunda línea y, a la vez, la técnica 
más difícil de implementar en Chile, tanto por la necesidad de contar con radiólogos especializados, como por el costo de los insumos. Pareciera razonable que esta técnica se hiciera exclusivamente en centros de referencia, públicos o privados. Llama sí la atención que nunca haya sido implementada en una IPU, lo que podría estar reflejando atraso tecnológico de ellas. Sorprende, y es de lamentar, que esta técnica se tenga presente y se pueda acceder a ella en sólo $26 \%$ de las IPU y que pareciera que no existen canales expeditos de derivación en la mayoría de las IPU para su eventual instalación fuera de ellas.

En ausencia de TIPS, la cirugía se transforma en las IPU en la única terapéutica factible de segunda línea, aunque aquí, una vez más, aparece la cirugía como una técnica más disponible en IPR que en las IPU. La explicación está muy probablemente en el deterioro que se produce en la función hepática en los pacientes que han sangrado persistentemente por VEG, lo que hace de la cirugía un tratamiento de excepción.

Por lo anterior, al fracasar la ligadura o la esclerosis, o al no acceder a endoscopia, en las IPU se instala habitualmente un balón de Sengstaken. Esta técnica, incómoda y asociada a importantes complicaciones, es un recurso frecuente en las IPU, lo que permite ocultar lo precario del tratamiento que se está ofreciendo. Esta falencia inevitablemente debe traducirse en una mayor mortalidad. De hecho, en una IPU se constató que los pacientes sangrantes por VEG que accedían a endoscopia diagnóstica y terapéutica, fallecían $6,7 \%$ versus $33 \%$ de los que sólo accedían a balones de Sengstaken ${ }^{5}$.

La política de erradicación de VEG, que alcanza $84 \%$ en las IPU y $94 \%$ en las IPR, es insuficiente en ambos grupos de instituciones ${ }^{2}$. La profilaxis del sangrado por VEG tampoco se hace en todos los establecimientos. La explicación puede buscarse en la falta de recursos en las IPU; pero no hay

\section{REFERENCIAS}

1. Kleber G, Sauerbruch T, Ansari H, Paumgartner G. Prediction of variceal hemorrhage in cirrhosis: A prospective follow-up study. Gastroenterology 1991; 100: $1332-7$. excusa para que tampoco se cumpla en todas las IPR. Llama la atención que las ligaduras elásticas se estén utilizando en diversas instituciones del país en la profilaxis de la primera hemorragia antes que la comunidad internacional valide esa conducta. Pareciera razonable que los gastroenterólogos chilenos hiciéramos un trabajo cooperativo evaluando en nuestro medio betabloqueadores y ligaduras. Por otro lado, la prevención del primer sangrado, así como el de la recidiva hemorrágica, son conductas mundialmente establecidas, por lo que también parece oportuno que las Sociedades Científicas en conjunto con la autoridad sanitaria insistan en la necesidad de cumplir conductas validadas en la comunidad internacional ${ }^{2}$.

El trabajo en el campo de las hemorragias digestivas, y en particular en várices esófago gástricas, tiene mucho que mejorar. Este estudio ha evidenciado una profunda inequidad sanitaria entre los grupos sociales que tienen protección de salud privada y los que la tienen dependiente del Estado. Es imperioso que la autoridad enfrente la desigualdad en el manejo diagnóstico y terapéutico que estamos ofreciendo en las instituciones públicas. Es necesario estimular estudios cooperativos que permitan mejorar los niveles tecnológicos y utilizar guías de trabajo y protocolos con posterior evaluación de resultados y costos. El trabajo ordenado y protocolizado en las instituciones públicas y privadas y en las instituciones universitarias irá en beneficio del desarrollo de políticas de salud más eficientes y solidarias y la mejor atención de los pacientes que sufren una patología frecuente en nuestra comunidad.

\section{Agradecimientos}

A los médicos de las diversas unidades de endoscopia que nos informaron resultados que incluían sus limitaciones.

2. De Franchis R. Evolving consensus in portal hypertension. Report of the Baveno IV Consensus Workshop on methodology of diagnosis and therapy in portal hypertension. J Hepatol 2005; 43: 167-76.

3. Chalasani N, Kahi C, Francois F, Pinto A, Marathe A, BINI E ET AL. Improved patient survival after acute 
variceal bleeding: A multicentric, cohort study. Am J Gastroenterol 2003; 98: 653-9.

4. Instituto Nacional de Estadísticas de Chile. www.ine.cl (Consultado el 23-4-2006).

5. Morales X, Ovalie L, Candia L, Agar S, Estay R, VARGAS L. Evaluación de la hemorragia digestiva alta en el Hospital San Juan de Dios: Un hospital de alta complejidad y de referencia del Servicio de Salud Metropolitano. Gastro Latinoamericana 2003; 14: 386.

6. Ministerio de Salud de Chile. www.minsal.cl (Consultado el 23-4-2006).

7. Vargas L, Morales X, Ovalue L, Estay R, Soto JR. Ligadura elástica complementada de esclerosis en el tratamiento de várices esofágicas. Experiencia en la hemorragia activa y en el tratamiento electivo. Rev Méd Chile 2001; 129: 1387-94.

8. Stiegmann G, Goff J, Michaletz-Onody P, Korula J, LIEBERMAn D, SAeEd Z et al. Endoscopic sclerotherapy as compared with endoscopic ligation for bleeding esophageal varices. N Engl J Med 1992; 326: 1527-32.

9. Vargas L, Ovalue L, Estay R, Sepúlveda P, Melia B, Soto JR. Hemorragia por várices esófago-gástricas. Experiencia con cianoacrilato y polidocanol en 68 pacientes con hemorragia activa. Rev Méd Chile 1999; 127: 685-92.

10. Silva G, Quera R, Fluxá F, Sanhueza E, Segovia R, BRAHM J ET AL. Octreótido, tratamiento endoscópico o ambos en la hemorragia variceal activa en pacientes cirróticos: estudio multicéntrico. Rev Méd Chile 2004; 132: 265-94.

11. Cooperative Spanish-French Group for the treatment of BLEEDING ESOPHAGEAL VARICES. Randomized controlled trial comparing terlipressin vs endoscopic sclerotherapy in the treatment of acute variceal bleeding and prevention of early rebleeding. Hepatology 1997; 26 (suppl): 249A.

12. YAN BM. Emergency management of bleeding esophageal varices: drugs, bands or sleep? Can J Gastroenterol 2006; 20: 165-70. 